\title{
Hospital dietetics: the dietician's needs
}

\section{By Carol Bateman, Royal Free Hospital, Pond Street, London NW2 i $Q G$}

The hospital dietician needs to have some influence on the food provided for all patients, so that all nutritional needs are met. She or he needs to spend time advising in-patients and out-patients on how they should manage their prescribed therapeutic diets and, although the dietician many not actually prescribe those diets, she or he often advises medical staff on suitable diet prescriptions. Within the hospital the dietician needs to see that there is an efficient system whereby the right therapeutic diet may reach the right patient and that each patient understands the principles of the diet prescribed and how to cope with it on discharge. Out-patients may need follow-up visits to reiterate advice or to give continuing support.

Where there are specialist units, whose patients need specialized dietetic advice, the dietician needs to be part of the team caring for the patient, and to be kept fully aware of all the developments in the patient's treatment. The hospital dietician therefore needs to have good working relations with medical, nursing, pharmacy, catering and other staff.

The dietician also needs to be involved in teaching nursing staff the principles of dietetics, and in teaching catering staff the basic principles and practice of diet therapy. It may also be useful for the dietician to be involved in teaching medical students something of the practicalities of dietetic treatment.

For hospital dietetics to succeed, the dietician must have time to carry out all these tasks. Unfortunately, dietetic departments in the National Health Service are grossly understaffed and unless this situation can be remedied, hospital dietetics in many Health Districts will fail to meet the expectations of medical staff and, indeed, the expectations of dieticians themselves.

Where dietetic resources are spread thinly the dietician needs to make quite sure that any clerical tasks which can be done by others are passed on, that time is not wasted by seeing queues of obese patients referred to the dietician by despairing doctors and that the limits of the work that can be done well with staff available are made quite clear to medical and administrative staff. It can be difficult for a dietician to refuse to do more work with a senior consultant, and medical staff should therefore make sure they take the dietetic needs of any new project into consideration in the planning stage, so that they do not later try to pressurize an already over-burdened dietetic department into taking on work which the dieticians are then not able to carry out. 